\title{
Sight restoration
}

\section{Pawan Sinha* and Richard Held}

Address: 46-4077, MIT Department of Brain and Cognitive Sciences, 77 Massachusetts Avenue, Cambridge, MA 02139

*Corresponding author: Pawan Sinha (psinha@mit.edu)

Fl000 Medicine Reports 2012, 4:17 (doi:10.3410/M4-17)

This is an open-access article distributed under the terms of the Creative Commons Attribution-Non Commercial License (http://creativecommons.org/licenses/by-nc/3.0/legalcode), which permits unrestricted use, distribution, and reproduction in any medium, provided the original work is properly cited. You may not use this work for commercial purposes.

The electronic version of this article is the complete one and can be found at: http://f $1000 . c o m / r e p o r t s / m / 4 / 17$

\begin{abstract}
Cases of sight onset after extended periods of congenital blindness provide windows into visual development and brain plasticity. Such cases are extremely rare in the developed world. Here, we make the argument that in meeting a public health challenge in the developing world, that of providing treatment to curably blind children, we have the opportunity to have a beneficial impact on science and society simultaneously. A recent initiative, Project Prakash, is motivated by these twin goals. We briefly describe this effort, some of its early results, and also the caveats that need to be kept in mind when interpreting the findings.
\end{abstract}

\section{The confluence of a humanitarian need and a scientific quest}

The WHO estimates that there are over 1.5 million blind children in the world, of whom nearly $90 \%$ live in the developing nations (WHO Factsheet 282, 2011 and [1]). Many are congenitally blind. The visual handicap, coupled with extreme poverty greatly compromises the children's quality of life; childhood mortality rates are greatly elevated (Sightsavers estimates that fewer than $50 \%$ of blind children in India survive to adulthood) and the vast majority of blind children are unable to get an education. Notably, much of this blindness (over 70\%) is preventable or treatable (figure 1 shows two common treatable conditions). Most children, however, never receive medical care because of financial hardship or lack of medical access. Helping such children is one of the most compelling humanitarian needs we confront today.

Help for blind children can take many forms. It might involve transcending the blindness through assistive devices such as technological aids (sonar equipped canes or audible GPS, for instance) and improved social integration. These are certainly worthy goals to aim for, but they require significant investment in infrastructure and major changes in societal attitudes that might be slow in coming. A more direct way of helping blind children, of course, is to provide them sight restoring surgeries. To the extent that these surgeries are successful in bringing some modicum of functional vision to the children, they can be effective in immediately improving children's life prospects. As a bonus, this intervention also opens up an unprecedented scientific opportunity to study a fundamental open question: How does the brain learn to extract meaning from sensory information?

As the newly-sighted children embark on the enterprise of learning to see, studying their visual development promises to yield insights into questions regarding object learning and brain plasticity. A particular strength of this approach is that it affords us an opportunity to continuously follow the development of visual skills and associated neural markers from before the sight restoration treatment to after. Furthermore, working with older children with newly initiated sight allows us to sidestep some of the operational difficulties that infant researchers have had to contend with, and that have limited the scope and complexity of developmental studies undertaken thus far.

\section{Accounts of late sight onset}

In popular imagination through the ages, the initiation of sight in a blind person is nothing less than a miracle. The rarity of such an event contributes to this perception. Indeed, an Italian ophthalmologist named Alberto Valvo 
Figure I. Two important causes of treatable congenital blindness in the developing world
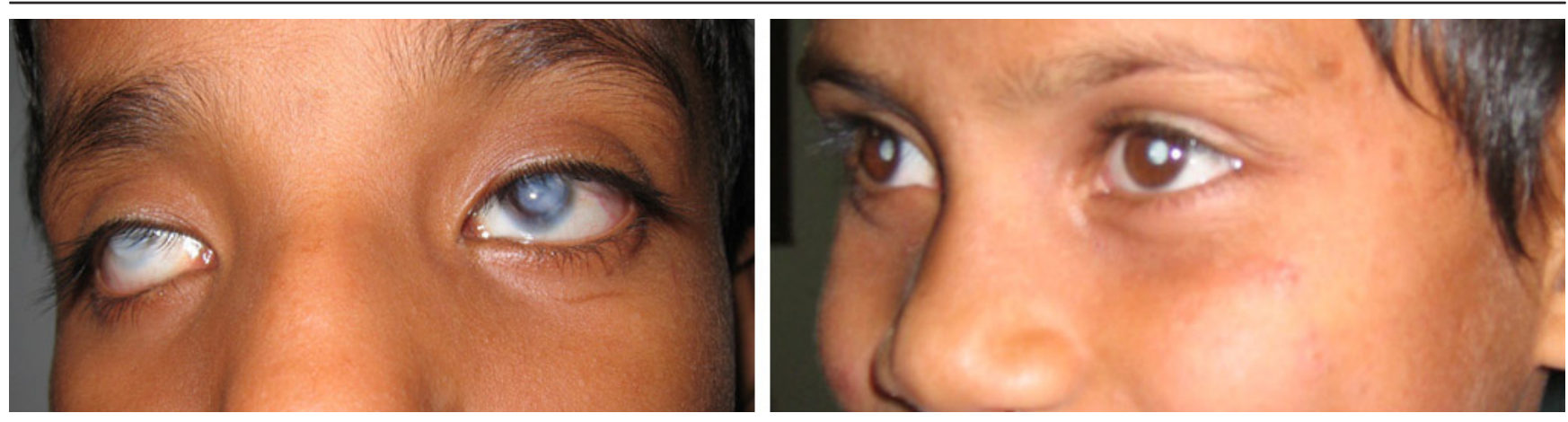

The left panel shows a child with bilateral corneal opacities and the right shows dense congenital cataracts. Both of these conditions are treatable with simple surgical procedures (grafting clear donor tissue for corneal opacities; lens extraction followed by intra-ocular lens implantation for cataracts). (Photographs by Pawan Sinha)

estimated that no more than 15 such cases had been described in any detail over the past millennium [2]. Just a handful more have been added to the list in the following four decades.

An English surgeon by the name of William Cheselden presented the first account of delayed sight onset in a 13 year old boy born with bilateral cataracts. Cheselden noticed that the boy had severely impaired visual abilities even after the removal of cataracts, as illustrated in this passage [3]:

"When he first saw, he was so far from making any judgment of distances, that he thought all object whatever touched his eyes (as he expressed it) as what he felt did his skin, and thought no object so agreeable as those which were smooth and regular, though he could form no judgment of their shape, or guess what it was in any object that was pleasing to him: he knew not the shape of anything, nor any one thing from another, however different in shape or magnitude; but upon being told what things were, whose form he knew before from feeling, he would carefully observe, that he might know them again."

However, it is difficult to cleanly interpret Cheselden's data since the child's struggles with acquiring visual proficiency could have been due either to central problems in learning or more peripheral issues of ocular healing, given the crude surgical techniques of that time, such as couching [4].

Modern literature on sight recovery in adulthood, following prolonged visual deprivation, is rather sparse [2,5-11], (see also [12] for a case of visual function recovery in adulthood, though not after blindness).
Maurer and her colleagues have found that brief periods of early deprivation, lasting just a few months, can have long-lasting impact on perception later in life. For instance, children who were treated for congenital cataracts within the first six months of life show subtle differences in processing facial configurations relative to their normally developing counterparts even after reaching teenage years [13]. As for instances of sight onset much later in life, the key findings from the few cases that have been reported thus far pertain to the difficulties the newly sighted experience in interpreting visual information as an ordered collection of meaningful entities. However, there is also evidence that these individuals over time are able to make effective use of visual information. For instance, Gregory and Wallace [6], in describing the patient denoted SB (who gained sight at the age of 52), write the following:

"SB had no difficulty in identifying the giraffe, elephant, monkey, lion, snakes or giant tortoises. He appeared to identify the tiger at once, though he was surprised to see that it was striped. ... At his favourite "locals" he cheered up considerably, and was clearly regarded as a "character". He was able to recognise his friends at a distance of at least fifteen feet, from one bar to another, and he would now cross roads with some confidence."

Similarly, Kurson [14] in his account of Mike May, who gained sight at the age of 45 , says the following:

"During lunch at the backyard patio table, May delighted in his ability to use vision alone to reach for the milk carton and find his napkin after it fell to the ground. He could easily distinguish between Burgess and Jennifer (May's wife and her friend) based on their hair lengths and the color of their clothes." 
Von Senden [5] reported that sight in adulthood was sometimes accompanied by emotional disturbances, perhaps in part due to the difficulties involved in accomplishing visual tasks that to others are so effortless. Carlson et al. [7] attribute some of the post-sight difficulties of their subject to motivational problems. As Gregory and Wallace write, in their account of SB:

"He certainly relied a great deal on vision, but we formed the impression that this very reliance cost him his selfrespect, for he had been proud of his abilities when the handicap was obvious, but now his previous achievements seemed paltry and his present position almost foolish. He was not a man to talk freely, but was obviously depressed."

Clearly, outcomes of sight-restoring surgeries late in life are complex, comprising both significant impairments as well as the development of important visual proficiencies. Given these complexities, a systematic research program with a larger subject population seems necessary. It is frustrating that most of the work thus far has been largely anecdotal in nature, a necessary consequence of the rarity of such cases.

A new initiative has begun to change this state of affairs by adding many more cases of late sight onset to the corpus thus far. Named Project Prakash (Sanskrit for light), this effort focuses on providing sight-restoring surgeries to congenitally blind children in rural India, the country with the largest population of blind children in the world. The project comprises an outreach program for identifying children with curable ocular pathologies, a medical treatment program for providing them surgical interventions and a schedule of post-operative follow-ups (www. ProjectPrakash.org; [15]). For each child, the Prakash studies include a clinical ophthalmic evaluation as well as assessments of various aspects of visual function. Figure 2 summarizes some of the tests employed.

The findings thus far suggest that, consistent with the notion of early sensitive periods for visual development, some key aspects of vision, such as acuity, spatial contrast sensitivity and precise oculomotor stability, are compromised by extended deprivation. The compromises appear to be permanent since the measures do not change significantly from soon after the surgery to even a year later.

Some encouraging results emerge when we look beyond these basic measurements towards 'higher-order' visual functions. Evidence thus far points to partial skill acquisition on tasks such as simple shape matching and face recognition $[16,17]$. Performance of the newly sighted on several of these tasks is found to be well above chance, even though it might not be as good as that of controls. While some of the residual performance deficits can be attributed to the subpar acuity and lingering nystagmus following surgery, others are harder to explain. For instance, newly sighted children tend to exhibit perceptual overfragmentation (a tendency to break up images into many little pieces, rather than wholistic objects) for several months following sight onset. This pattern of proficiencies and limitations may help us better characterize the limits of neural plasticity late in childhood. In clarifying the prospects of visual function acquisition after many years of early blindness, these results hold significance for basic neuroscience as well as the practice of pediatric ophthalmology and the implementation of late stage blindness treatment programs.

Moving beyond the mere demonstration of visual recovery, longitudinal studies in the Prakash population, are beginning to provide clues regarding the process of recovery [18]. The elucidation of this arc of development opens up the possibility of mechanistically specifying how visual bootstrapping happens and how complex visual skills derive from simpler ones that precede them in developmental chronology. Along these lines, recent results have highlighted the profound significance of dynamic information processing as a building block for other visual skills.

Another fundamental issue that these studies can help address is that of cross-modal mapping - correlating visual information with those from other sensory modalities, such as touch and audition. Is this linkage established via innate 'amodal' processes or via a process of learning? This foundational question, which was framed over three centuries ago by philosophers such as John Locke and William Molyneux, is central to understanding the nature of internal representations and the nativism versus empiricism debate [19]. The question has remained tantalizingly open ever since its formulation. Studies with the newly sighted have finally begun outlining an answer [20]. After showing no evidence of cross-modal transfer immediately after surgery, newly sighted children achieve proficiency in periods as short as a week. This points to the existence of rapid learning processes for detecting relationships across sensory streams, a result that can help seed important theoretical work on learning algorithms in the brain.

Taken together, these findings are beginning to suggest that the landscape of plasticity late in the developmental timeline is likely to be complex. Neither of the two extreme positions appear tenable; on the one hand, not all visual functions seem subject to strict early critical 
Figure 2. Some of the tests included in the Prakash assessment battery

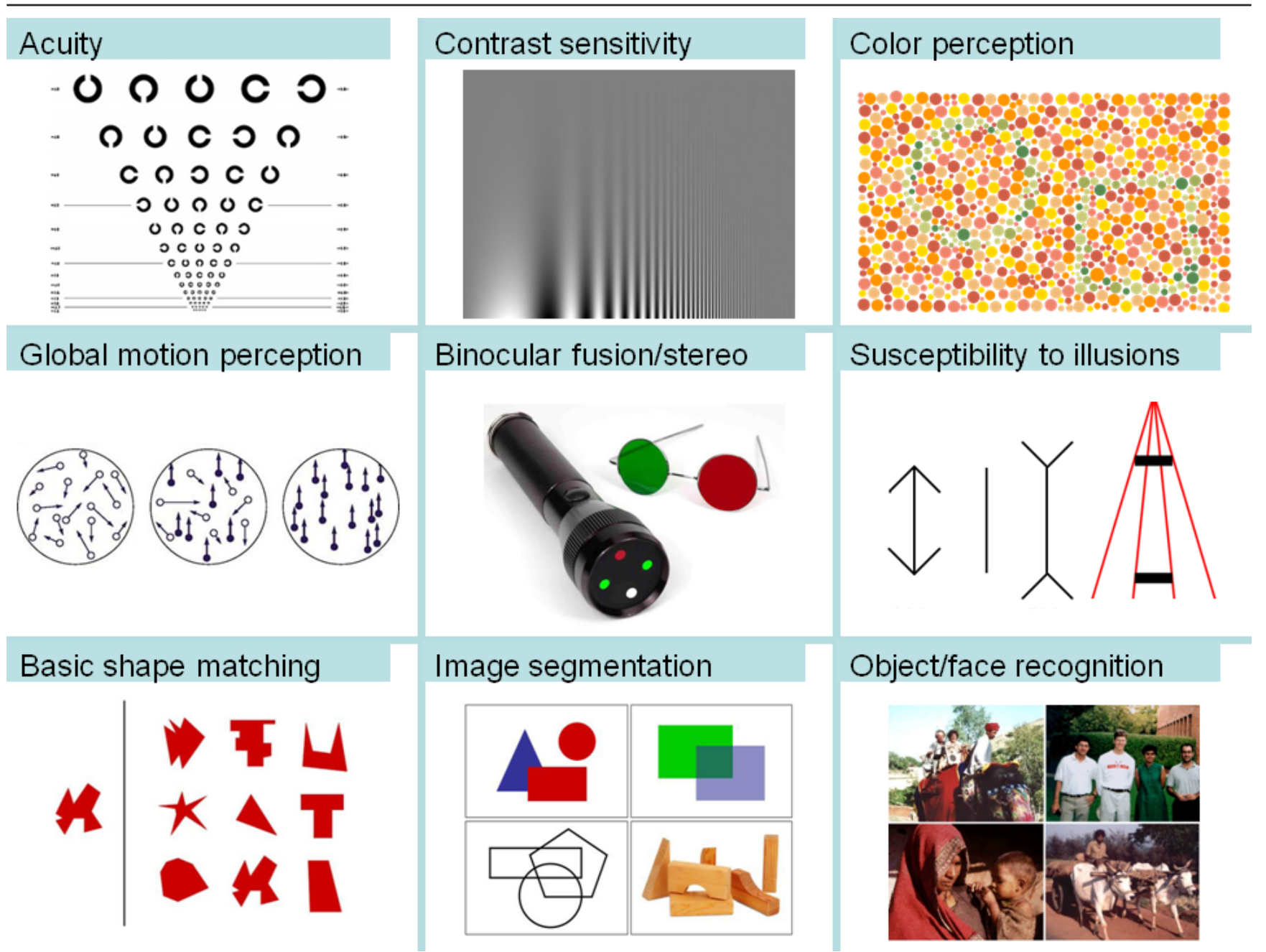

Besides basic measurements of acuity, contrast sensitivity and motion coherence thresholds, the tests for examining visual function and its development following late sight-onset also assess children's performance on 'higher-order' visual tasks such as image segmentation, shape matching and face localization. (Image credits: Landolt C chart from Precision Vision Inc. and the Worth Four dot test from Optimetrics Inc.)

periods and, on the other hand, early visual experience is undeniably important for the normal development of key abilities like high-resolution vision. Individuals with late sight onset seem to possess the basic substrates for at least some aspects of visual learning, although the eventual extent of their proficiency might be limited by the deprivation they experienced early in their developmental trajectories. This pattern of results suggests a fascinating interplay of nature and nurture processes in visual development and points to a wealth of questions to be tackled in the years to come.

\section{Reasons for caution}

Studies of newly sighted individuals promise many insights regarding visual learning. However, it is important that we do not over-interpret the data or misconstrue their relationships with other issues in neuroscience. There are at least two questions that give us reasons for caution.

\section{Can studies of vision following late sight onset help us} understand the mechanisms of normal visual development? A sight-restored child is not a talking infant. We cannot assume a priori that the developmental trajectory following late sight onset will recapitulate in whole or in part the normal progression of visual development in a newborn. Even though the two children, one normally sighted and the other late-sighted, might reach similar levels of visual proficiency on a given task, they may do so by following distinct developmental paths. There are several reasons 
why the paths might deviate relative to each other. Visual learning in a newly sighted adolescent, for instance, can ride atop the world model that has already been put in place by other modalities. The child already knows about the world as being made up of distinct objects, has linguistic labels for different categories and has in place concepts such as object permanence and solidity. These pre-existing notions might facilitate the development of visual object concepts in the late-sighted. An infant, by contrast, starts with a more rudimentary notion of the composition of the world. Additionally, an older child who is just beginning to see has much greater abilities for interacting with, and actively manipulating, the world relative to an infant. This richness of interaction may modify the rapidity and manner in which visual concepts are acquired. Finally, from a purely physiological perspective, the brain of an older child differs markedly from that of an infant. The processes of neuronal migration, axonal myelinization and dendritic pruning which are so extensive in the infant brain [21] have largely run their course by adolescence. These physiological differences may impact developmental trajectories in complex ways and render visual learning in normally sighted infants to be quite different from that in older children with late sight onset.

For these reasons and several others, studies of late sight onset should not be construed as replacements for investigations of normal visual development. This does not in any way diminish the significance of studies of late sight onset; they are still invaluable as indicators of $a$ path towards visual proficiency. All we need to remember is that this path may not be the same as the one the brain follows in normal development. It is an interesting challenge to determine the extent to which the two kinds of trajectories actually share commonalities.

\section{What is the relationship between the new studies of late sight onset and previous animal experiments on critical periods?}

It is tempting to think of instances of visual learning after late sight onset as counterpoints to classical notions of critical periods in vision [22]. However, the linkage between the two bodies of work is not entirely straightforward. Even setting aside the fact that one body of literature arises from non-human animal studies, while the other is based on work with humans, there are several reasons why one has to exercise caution in relating these two lines of work. First, many, if not most, of the classical studies of visual critical periods have employed monocular deprivation protocols wherein one eye is sutured shut while the other eye has a normal visual experience [23-25]. Doing so sets up a strong competitive interaction between the two eyes with dramatic consequences on cortical organization.
However, the cases of late sight onset in humans profiled above involve roughly equal bilateral deprivation. Given the reduced competitive effects in the latter case, it cannot be assumed that its cortical consequences (both in terms of their nature and permanence) will be comparable to those that arise from monocular deprivation regimens. Furthermore, several previous studies of binocular deprivation have typically used manipulations that are not precisely analogous to the deprivation experienced by humans prior to gaining sight. Specifically, dark-rearing and lid-suture lead to a more impoverished visual experience than do cataracts and corneal opacities (the two primary conditions treated in humans). The latter allow greater light perception and even some rudimentary sense of the movement of shadows of objects close to the face. Accordingly, outcomes after these different kinds of blindness may well differ.

Some methodological issues are worth highlighting as well. Since traditional studies of critical periods were based on work with animal subjects, the complexity of the assessment techniques was necessarily limited; typically, the dependent variables were physiological, such as number of cells responding to one or the other eye. Behaviorally, while basic visual attributes like acuity and visuo-motor coordination can be measured (e.g. [26]), performance on high-level tasks such as object recognition and image segmentation is largely unknown. This makes it challenging to compare the two datasets, one from nonhuman subjects and the other from humans, that have employed non-identical sets of assessment tasks. Indeed, it appears that while visual deprivation in humans does permanently compromise acuity, consistent with classical studies with animals and the notion of critical periods, some higher-level visual skills can be acquired.

Given all of these considerations, it is clear that the older animal studies of deprived rearing differ in some important ways from reports of late sight onset in humans. Therefore, evidence of some visual skill acquisition in the latter ought not to be seen as a counterpoint to the notion of critical periods developed in the former. More broadly, the notion of critical periods is worthy of a fresh look. Even within the body of animal deprivation studies, results of the consequences of early deprivation are not entirely homogenous; several studies have reported significant gains in visual abilities over time after deprivation's end [27-32]. The reasons for these disparities in outcomes are not entirely clear.

\section{Looking ahead}

Studies of sight-restoration in children and adults have already yielded some important insights regarding the possibility of visual learning late in life, and the mechanisms 
that might underlie such learning. Further development of this research program will enable us to address several additional major questions that have hitherto resisted systematic empirical investigation.

A prominent open question ripe for inquiry relates to changes in cortical functional organization as assessed via non-invasive brain imaging techniques. How does the cortex reorganize after sight-onset? Specifically, is there cross-modal activation in the visual cortex prior to surgery (e.g. [33]) that dissipates with sight-onset? To what extent can the influx of visual information late in life undo the oft-reported co-option of the visual cortex by other sensory modalities in the blind [34]? Studying the behavioral changes, if any, in sensory sensitivities from before to after sight onset and correlating them with observed cortical changes can allow us to make inferences regarding the functional consequences of cortical reorganization.

Future studies of sight restoration can also help refine the notion of critical periods. Classical animal studies of impoverished rearing have yielded evidence for sensitive periods in visual development. Yet, as we described in the foregoing section, there are several where caution needs to be exercised when extrapolating those results to cases of late sight onset in humans. We need to get empirical data from human subjects that can directly relate extent of deprivation to quality of post-operative vision. Such data will also enable us to build a taxonomy of visual skills according to their susceptibility to early deprivation. A blanket notion of 'critical periods' is perhaps too broad to apply to the entire gamut of visual functions. For instance, while acuity might be highly sensitive to early deprivation, does binocular depth perception follow similar rules? Are some aspects of high-level vision, say face recognition and visual imagery, more sensitive to early visual histories than others? From this body of data, we will be able to begin constructing an understanding of the rules of visual plasticity and why some abilities might be permanently compromised due to an initial period of blindness while others might be spared. In this context, interesting connections wait to be drawn between behavioral findings of spared or compromised visual abilities on the one hand and recent work on molecular mechanisms related to neural plasticity [35], on the other.

From an applied perspective too, studies of sightrestoration promise to yield rich dividends. The development of interventions and therapies for improving visual function in the newly sighted can have relevance for the broader domain of visual disorders including such conditions as amblyopia and even some neurological ones like autism which are reported to have strong visual correlates [36]. The interactions might well be bi-directional; treatments developed for amblyopia might help improve low-level visual outcomes in the newly sighted children.

The research program of providing sight to the blind and studying their subsequent visual progress is distinguished by its far-reaching implications from both fundamental and applied perspectives. It is an excellent example of a win-win partnership between the medical and scientific worlds and can be expected to yield many foundational clues about how the brain comes to extract meaning from sensory inputs.

\section{Competing interest}

The authors declare that they have no competing interests.

\section{Acknowledgments}

The authors' work on sight restoration and visual learning is supported by grants from the National Eye Institute (NIH grant R01EY020517), the James McDonnell Foundation and the Simons Foundation.

\section{References}

I. Parikshit G, Clare G: Blindness in children: a worldwide perspective. Community Eye Health 2007, 20:32-3.

2. Valvo A: Sight restoration after long-term blindness: The problems and behavior patterns of visual rehabilitation. New York: American Foundation for the Blind; 197I.

3. Cheselden W: An Account of Some Observations Made by a Young Gentleman, Who Was Born Blind, or Lost His Sight so Early, That He Had no Remembrance of Ever Having Seen, and Was Couch'd between 13 and 14 Years of Age. Philosophical Transactions 1727, 35:447-50.

4. Prioreschi P: A History of Medicine. Vol. I, 2nd ed. Omaha, Nebraska: Horatius Press; 1996.

5. Von Senden M: Space and Sight: The Perception of Space and Shape in the Congenitally Blind Before and After Operation. Reprint, Glencoe, IL: Free Press; 1960.

6. Gregory RL, Wallace JG: Recovery from early blindness: A case study. Quarterly Journal of Psychology. Monograph no. 2. 1963.

7. Carlson S, Hyvärinen L, Raninen A: Persistent behavioural blindness after early visual deprivation and active visual rehabilitation: a case report. Br J Ophthalmol 1986, 70:607-II.

8. Sacks O: To see and not see. In An Anthropologist on Mars, Vintage Books, New York: Alfred A. Knopf; 1995:108-I52.

9. Fine I, Wade AR, Brewer AA, May MG, Goodman DF, Boynton GM, Wandell BA, MacLeod DIA: Long-term deprivation affects visual perception and cortex. Nat Neurosci 2003, 6:915-6.

FI000 Factor 8

Vivien Casagrande 09 Oct 2003

10. Maurer D, Lewis TL, Mondloch CJ: Missing sights: consequences for visual cognitive development. Trends Cogn Sci (Regul Ed) 2005, 9:|44-5|

II. Levin N, Dumoulin SO, Winawer J, Dougherty RF, Wandell BA: Cortical maps and white matter tracts following long period of visual deprivation and retinal image restoration. Neuron 20|0, 65:2|-3|.

12. Barry SR: Fixing My Gaze: A Scientist's Journey into Seeing in Three Dimensions. Basic Books, New York; 2009. 
13. Le Grand R, Mondloch CJ, Maurer D, Brent HP: Neuroperception. Early visual experience and face processing. Nature 200I, 410:890.

FI000 Factor 6

Pawan Sinha 16 Aug 2012

14. Kurson R: Crashing Through: A True Story of Risk, Adventure, and the Man Who Dared to See. Reed Elsevier Inc; 2007.

15. Mandavilli A: Visual neuroscience: look and learn. Nature 2006, 44I:27I-2.

16. Bouvrie JV, Sinha P: Visual object concept discovery: Observations in congenitally blind children, and a computational approach. Neurocomputing 2007, 70:2218-33.

17. Ostrovsky $Y$, Andalman A, Sinha P: Vision following extended congenital blindness. Psychol Sci 2006, 17:1009-14.

18. Ostrovsky Y, Meyers E, Ganesh S, Mathur U, Sinha P: Visual parsing after recovery from blindness. Psychol Sci 2009, 20:|484-9|.

FI000 Factor 8

Jitendra Sharma, Hiroki Sugihara and Scott Gorlin 10 Feb 2011

19. Morgan M: Molyneux's Question. Cambridge Cambridge: University Press; 1977.

20. Held R, Ostrovsky Y, Gelder B de, deGelder B, Gandhi T, Ganesh S, Mathur $U$, Sinha $P$ : The newly sighted fail to match seen with felt. Nat Neurosci 20II, 14:55I-3.

FI000 Factor 12

Melvyn Goodale and Gavin Buckingham 20 May 201I, Jitendra Sharma and Hiroki Sugihara 26 Oct 20II

21. Kolb B, Whishaw IQ: Fundamentals of Human Neuropsychology. Macmillan; 2008.

22. Daw NW: Critical Periods in the Visual System. In Neurobiology of Infant Vision, Edited by Hopkins B, Johnson SP. Westport: Praeger; 2003.

23. Hubel DH, Wiesel TN: Effects Of Monocular Deprivation In Kittens. Naunyn Schmiedebergs Arch Exp Pathol Pharmakol 1964, 248:492-7.

FI000 Factor 6

Pawan Sinha 16 Aug 2012

24. Wiesel TN, Hubel DH: Extent of recovery from the effects of visual deprivation in kittens. J Neurophysiol 1965, 28:1060-72.
25. Lehmkuhle S, Kratz KE, Sherman SM: A behavioral inventory of the spatial sensitivity of visually deprived cats. Invest Ophthalmol Vis Sci 198I, Suppl, 20-48.

26. Smith DC, Lorber R, Stanford LR, Loop MS: Visual acuity following binocular deprivation in the cat. Brain Res 1980, I83:I-II.

27. van Hof-Van Duin J: Development of visuomotor behavior in normal and dark-reared cats. Brain Res 1976, 104:233-4I.

28. Timney B, Mitchell DE, Giffin F: The development of vision in cats after extended periods of dark-rearing. Exp Brain Res 1978, 3I:547-60.

29. Kaye M, Mitchell DE, Cynader M: Depth perception, eye alignment and cortical ocular dominance of dark-related cats. Brain Res 198I, 254:37-53.

30. Mower GD, Caplan C], Letsou G: Behavioral recovery from binocular deprivation in the cat. Behav Brain Res 1982, 4:209-15.

31. Mitchell DE: The extent of visual recovery from early monocular or binocular visual deprivation in kittens. J Physiol (Lond) 1988, 395:639-60.

FI000 Factor 6

Pawan Sinha 16 Aug 2012

32. Zabłocka $T$, Zernicki B: Binocularly deprived cats are normal in visual discrimination learning in a simple apparatus. Acta Neurobiol Exp (Wars) 1988, 48:215-21.

33. Merabet LB, Pascual-Leone A: Neural reorganization following sensory loss: the opportunity of change. Nat Rev Neurosci 2010 , I I:44-52.

FI000 Factor 6

Pawan Sinha 16 Aug 2012

34. Burton $\mathrm{H}$ : Visual cortex activity in early and late blind people. J Neurosci 2003, 23:4005-II.

FI000 Factor 6

Pawan Sinha 16 Aug 2012

35. Bavelier D, Levi DM, Li RW, Dan Y, Hensch TK: Removing brakes on adult brain plasticity: from molecular to behavioral interventions. J Neurosci 2010, 30:|4964-7|.

36. Behrmann M, Thomas C, Humphreys K: Seeing it differently: visual processing in autism. Trends Cogn Sci (Regul Ed) 2006, 10:258-64. 